\title{
Micromechanical Modeling of the Banded Structure of Martensite in Small Inclusions
}

\author{
G. Reisner and F.D. Fischer \\ Institute of Mechanics, Montanuniversität Leoben, 8700 Leoben, Austria
}

\begin{abstract}
The stability of austenite against strain induced martensitic transformation (SIMT) strongly depends on the grain size of the austenite. This is observed in austenitic steels with grain sizes from several $\mu m$ up to several hundred $\mu m$ and for much smaller austenitic grains (three orders of magnitude) that are embedded in a non austenitic matrix. $f c c$ Fe-precipitates may be formed in dilute $\mathrm{Cu}-\mathrm{Fe}$ alloys by a certain heat treatment. These Fe-particles transform only by plastic deformation of the alloy. The SIMT leads to a banded structure with parallel layers of martensite. We investigate the influence of the external stress state on the microstructure (number of twin bands, relative thickness of the bands) with the help of a micromechanical model. The grain size effect on the austenite's stability against SIMT and on the microstructure may be explained by a thermodynamic transformation criterion and the local mechanical driving force.
\end{abstract}

\section{INTRODUCTION}

Several researchers studied experimentally the strain induced martensitic transformation (SIMT) in dilute $\mathrm{Cu}-\mathrm{Fe}$ alloys [1-6]. Due to coherency strains $f c c$ Fe-precipitates may be grown from supersaturated $\mathrm{Cu}$ mono- or polycrystals. The Fe-precipitates remain austenitic on cooling to room temperature, but transform martensitically into a banded structure on plastic deformation of the material [6-8]. The interaction of two dislocations on distinct slip planes within the fcc Fe particle was determined to be the triggering mechanism for the martensitic transformation [8]. Ishikawa also reports that the martensite is internally-twinned [8]. Particles with a radius less than $10 \mathrm{~nm}$ are fully coherent with the Cu-matrix. If the radius is larger than $10 \mathrm{~nm}$, the particles become semi-coherent or fully incoherent [7]. The size effect on the stability against the martensitic transformation has been studied considering the elastic energy only [6]. Since the martensitic transformation causes a volume dilatation of $3.6 \%$, the material surrounding the Fe-particles has to yield to accommodate the shape change. Even if yielding occurs only in a small region it reduces the elastic strain energy of the whole body. Considering a purely elastic deformation thus may not be sufficient. This motivates a finite element simulation that allows to calculate the stress and strain states also in an elastic-plastic material.

\section{GEOMETRICAL AND MATERIAL MODEL}

For our simulation we choose a representative volume element (RVE) with a single austenitic inclusion embedded in a Cu-matrix. The volume fraction of the inclusion is $2 \%$. For the sake of simplicity we consider a thin slab of the alloy so that the part of the spherical inclusion inside the RVE can be approximated by a cylinder. To account for this simplification of the geometry, generalized plane strain conditions are prescribed with respect to the bottom and top plane of the slab [9]. Assuming a hexagonal pattern of inclusions, the slab can be divided into hexagonal prisms each with a $\mathrm{Fe}$ precipitate in its center (Fig. 1). Periodic boundary conditions are enforced on the surface of the unit cell. 


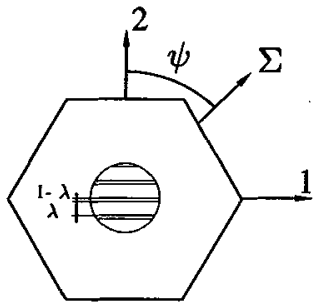

Figure 1: Sketch of the representative volume element (RVE) of the CuFe alloy. The volume fraction of the $f c c$ Fe-precipitate is $2 \%$ and the number of martensite bands is 8 . The austenite inclusion is exaggerated in size. The direction 3 is perpendicular to the plane. $\psi$ denotes the angle between the direction of $\Sigma$ and direction 2 . The relative band thickness is denoted by $\lambda$.

The cylinder is divided into several parallel slabs. Each layer corresponds to a martensite band. The thickness ratio of two neighboring slabs is given by $\frac{\lambda}{1-\lambda}$ where $\lambda$ denotes the relative band thickness. As depicted in Fig. 1, direction 1 is parallel and direction 2 is normal to the interface of two neighboring martensite bands.

We consider two distinct loading cases, namely a uniaxial tensile test and a single pass cold rolling process. In both cases the applied force acts in the transversal plane (i.e. the plane given by directions 1 and 2) of the RVE. The single pass cold rolling process is modeled by a plane strain compressive test to account for the plane deformation in the rolling process.

Similar to a self consistent approach the experimental flow curve of a $\mathrm{Cu}-2 \% \mathrm{Fe}$ alloy with a mean Fe-particle diameter of $40 \mathrm{~nm}$ as reported by Tiefenthaler et al. is assigned to the Cu-matrix [10]. We assume that the flow behavior of the single crystal Fe-precipitates in both the austenitic and martensitic states does not change with the particle size. For all phases a $J_{2}$ flow theory, isotropic hardening and a Ludwik-type of hardening behavior are assumed [11]:

$$
\Sigma=\Sigma_{0}+K \varphi^{n}
$$

The smallest transformable volume is a single layer. If the transformation criterion is fulfilled in a certain layer, the martensitic transformation is simulated by attaching the stress free transformation strain $\underline{\epsilon}^{\mathrm{T}}$ to $V_{\mu}$. During the transformation the external load is held constant. Following the phenomenological theory of the martensitic transformation by Wechsler, Lieberman and Read (WLR), the deformation gradient can be determined solely from the crystall structure and the lattice parameters of the parent and the product phase [12]. We use a small strain approximation to obtain $\underline{\underline{\epsilon}}^{\mathrm{T}}$. The lattice parameter $a_{0}$ of the $f c c$ parent phase austenite is $0.361 \mathrm{~nm}$. Due to the small amount of $\mathrm{Cu}$ solved in the Fe-precipitate the product phase martensite shows a bct lattice with a ratio of tetragonality of $\frac{c}{a} \simeq 1.1$ (lattice parameters $a=0.28$ and $c=0.31 \mathrm{~nm}$ ) [13]. The volume dilatation of $\delta=3.6 \%$ is achieved by an expansion normal to the habit plane, only. The shear deformation of $\gamma_{0}=2 \epsilon_{12}^{T}=0.156$ acts in the $1-2$ plane. Neighboring layers of martensite shear into opposite directions.

\section{TRANSFORMATION CRITERION}

In this report, we apply a local transformation criterion (LTC) proposed by Fischer [14] for the transformation of a certain microregion $V_{\mu}$ : 
Table 1: Material input data for the numerical simulation of SIMT in a dilute Cu-2\%Fe alloy. A Ludwik-type of flow behavior is assumed. $\Sigma_{0}$ denotes the yield strength. $K$ and $n$ are hardening parameters and $\nu$ is the Possion's ratio.

\begin{tabular}{|c|c|c|c|c|c|}
\hline Phase & $\Sigma_{0}[\mathrm{MPa}]$ & $K[\mathrm{MPa}]$ & $n$ & $\nu$ & Young's modulus [MPa] \\
\hline Copper & 120 & 432 & 0.46 & 0.28 & 130.000 \\
\hline Austenite & 200 & 300 & 0.60 & 0.3 & 210.000 \\
\hline Martensite & 400 & 150 & 0.80 & 0.3 & 210.000 \\
\hline
\end{tabular}

$$
\int_{V_{\mu}}\left(\underline{\underline{\Sigma}}+\underline{\underline{\sigma}}^{\mathrm{int}}\right): \underline{\underline{\epsilon}}^{\mathrm{T}} d V+\int_{V_{\mu}} \rho \Delta \varphi_{\text {chem }} d V=F_{\mathrm{c}}+\Delta \Gamma+\int_{V} \int_{0}^{t} \underline{\underline{\tau}}: \underline{\underline{\epsilon}}^{\mathrm{pl}} d \tilde{t} d V+\frac{1}{2} \int_{V} \underline{\underline{\tau}}: \underline{\underline{\underline{L}}}^{-1}: \underline{\underline{\tau}} d V .
$$

The local stress state $\underline{\underline{\sigma}}$ can be expressed as the sum of the external stress state $\underline{\underline{\Sigma}}$, an internal stress state $\underline{\underline{\sigma}}^{\text {int }}$ due to the stress redistribution caused by the microstructure and an additonal internal stress state $\underline{\underline{\tau}}$ that represents the stress fluctuation introduced by the martensitic transformation of $V_{\mu}\left(\underline{\underline{\sigma}}=\underline{\underline{\underline{\Sigma}}}+\underline{\underline{\underline{\sigma}}}^{\text {int }}+\underline{\underline{\tau}}, \int_{V} \underline{\underline{\sigma}}^{\text {int }} d V=\int_{V} \underline{\underline{\tau}} d V=\underline{\underline{0}}\right)$. The stress free transformation strain $\underline{\underline{\epsilon}}^{\mathrm{T}}$ is only present in the transforming microregion $V_{\mu} \cdot \int_{V_{\mu}} \underline{\underline{\sigma}}: \underline{\underline{\epsilon}}^{\mathrm{T}} d V$ denotes the mechanical driving force $(M D F)$. Patel and Cohen introduced the term mechanical driving force already 40 years ago in their pioneering work [15]. However, in their terminology the $M D F$ is the product of the external stress state with the stress free transformation strain $\left(M D F=\underline{\underline{\Sigma}}: \underline{\underline{\epsilon}}^{\mathrm{T}}\right)$. The chemical driving force $(C D F)$ is understood as the difference of the chemical energy of the stress free phases austenite and martensite, $\Delta \varphi_{\text {chem }}$, weighted by the density $\rho$ integrated over the transforming region $V_{\mu}$.

The terms on the right hand side of Eq. 2 represent different contributions to the transformation barrier. $\Delta \Gamma$ stands for the change in energy due to the newly formed interfaces and depends on the particle radius and the specific energies of the different interfaces. $F_{\mathbf{c}}$ is the critical thermodynamic force necessary to move the austenite - martensite interface. It is interesting to note that not the total plastic dissipation $\left(\iint_{0}^{t} \underline{\underline{\sigma}}: \underline{\underline{\epsilon}}^{\mathrm{pl}} d \tilde{t} d V\right)$ due to the martensitic transformation acts as restricting force but only the plastic dissipation due to the fluctuation of the stress state $(\underline{\underline{\tau}})$. This part of the plastic dissipation $\int_{V}^{t} \int_{0}^{t} \underline{\underline{\tau}}: \frac{\dot{\epsilon}}{\mathrm{pl}}^{\mathrm{pl}} d \tilde{t} d V$ will be referred to as $W_{\mathrm{pl}}^{\tau}$. The integration with respect to time in the above expression of $W_{\mathrm{pl}}^{\tau}$ indicates the path dependence of the plastic dissipation. The transformation of the microregion $V_{\mu}$ takes place in the time range from 0 to $t$. However, $t$ only has the meaning of a process parameter. With $\underline{\underline{\underline{\underline{L}}}}$ being the elasticity tensor, $\frac{1}{2} \int_{V} \underline{\underline{\tau}}: \underline{\underline{\underline{\underline{L}}}}^{-1}: \underline{\underline{\tau}} d V$ yields the strain energy solely due to $\underline{\underline{\tau}}$ and is denoted by $W_{\mathrm{el}}^{\tau}$.

The specific interface energy of a habit plane is estimated as $0.02[\mathrm{~N} / \mathrm{m}]$. This value is larger than the one for a coherent twin plane in austenite $(0.013 \mathrm{~N} / \mathrm{m})$ and smaller than the one for a $\gamma / \bar{\gamma}$ large angle grain boundary, which can be estimated as four times as large as twin plane energy [16]. The specific energy of a martensite to Cu-matrix interface is assumed to be $0.2[\mathrm{~N} / \mathrm{m}]$ larger than the specific energy of the austenite/copper interface. Since the area of the interfaces scales with $r^{2}$ and $V_{\mu}$ scales with $r^{3}$ (particle radius $r$ ), the interface energy per $V_{\mu}, \Delta \Gamma^{\prime}$, is proportional to $\frac{1}{r}$. This is the reason for the importance of $\Delta \Gamma^{\prime}$ in the transformation barrier for the very small Fe-precipitates in the $\mathrm{Cu}-\mathrm{Fe}$ alloy. From Fig. 2 one can see that $\Delta \Gamma^{\prime}$ takes a minimum for $N=5$, irrespective of the particle size $r$.

The specific values $\left(W_{\mathrm{el}}^{\tau}\right)^{\prime}$ and $\left(W_{\mathrm{pl}}^{\tau}\right)^{\prime}$ are determined from numerical simulations in which the

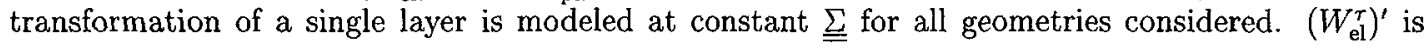



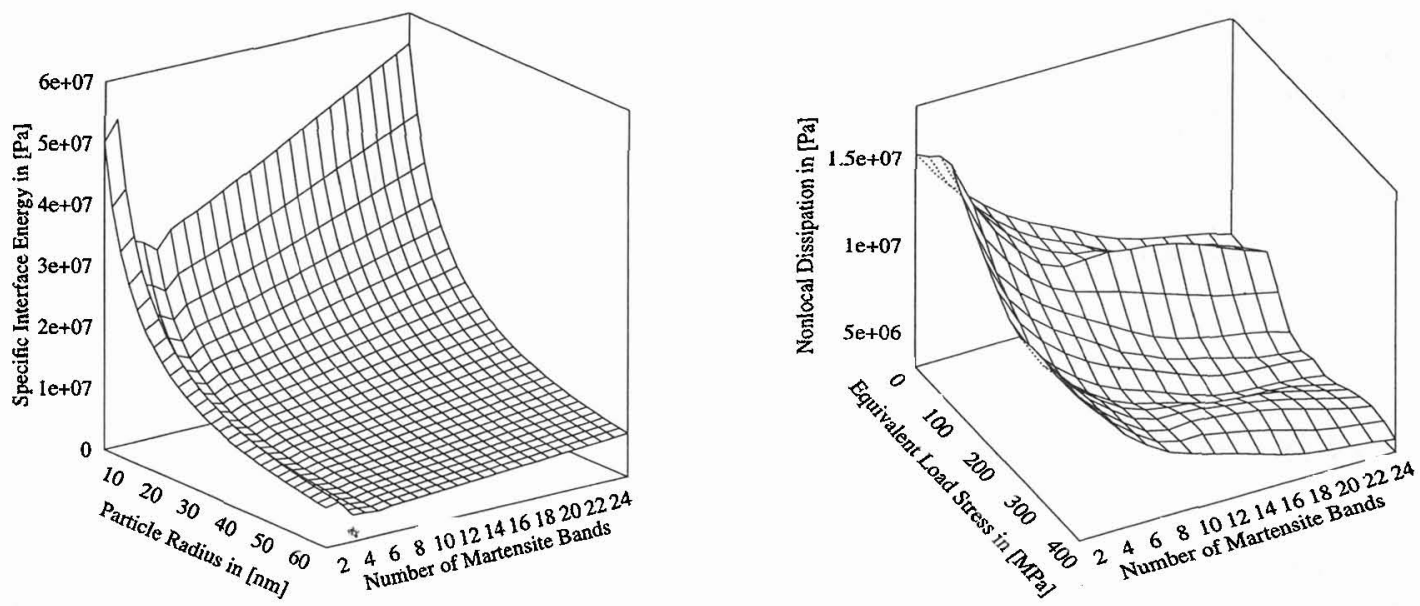

Figure 2: (Left) The specific interface energy $\Delta \Gamma^{\prime}$ per unit volume of the transforming material as a function of the particle radius $r$ and the number of martensite bands $N$. (Right) Nonlocal plastic dissipation $\left(W_{\mathrm{pl}}^{\tau}\right)^{\prime}$ due to the stress fluctuations $\underline{\tau}$ caused by SIMT of the first layer that transforms as function of the external stress $\underline{\underline{\Sigma}}$ and the number of martensite bands.

relatively small as compared to $\left(W_{\mathrm{pl}}^{\tau}\right)^{\prime}$ and is thus neglected. Fig. 2 shows $\left(W_{\mathrm{pl}}^{\tau}\right)^{\prime}$ as a function of $N$ and the equivalent external stress $\underline{\underline{\Sigma}}$. Forming many martensitic twin bands reduces the overall shape change of the inclusion and thus necessitates less plastic accommodation. $\left(W_{\mathrm{pl}}^{\tau}\right)^{\prime}$ depends on $\underline{\underline{\Sigma}}$, since the hardening behavior of the materials is nonlinear (i.e. the higher $\underline{\underline{\Sigma}}$, the smaller is the stress increase due to $\underline{\underline{\epsilon}}^{\mathrm{T}}$ and hence also $\left.\left(W_{\mathrm{pl}}^{\tau}\right)^{\prime}\right)$.

The stability of the Fe-precipitates in a Cu-matrix against SIMT depends on the particle size. The martensite start temperature $M_{s}$ can be regarded as a measure for this stability. Li et al. [7] reported that particles of typically several hundred nanometers in diameter loose their stability at room temperature $(300 \mathrm{~K})$ and transform to martensite without any external load. However, smaller precipitates, even if they are completely incoherent with the Cu-matrix, remain austenitic at room temperature [7]. Applying the LTC given by Eq. 2 for the case that particles of radius $R \approx 250 \mathrm{~nm}$ transform at room temperature in the absence of an applied load $\left(M D F^{\prime}=0\right)$ we find:

$$
C D F^{\prime}=F_{\mathrm{c}}^{\prime}+\left.\left(\Delta \Gamma^{\prime}+\left(W_{\mathrm{pl}}^{\dot{\tau}}\right)^{\prime}\right)\right|_{\mathrm{R} \sim 250 \mathrm{~nm}} .
$$

In the following, it is assumed that $C D F^{\prime}$ and $F_{\mathrm{c}}^{\prime}$ do not change with the particle size. At room temperature SIMT will occur in particles with $r<\mathrm{R}$, if an additional mechanical driving force is equivalent to the difference in the transformation barriers for the two particle sizes $r$ and $R$ :

$$
\left.M D F^{\prime}\right|_{300 \mathrm{~K}}=\left.\left(\Delta \Gamma^{\prime}+\left(W_{\mathrm{pl}}^{\tau}\right)^{\prime}\right)\right|_{r}-\left(\Delta \Gamma^{\prime}+\left.\left(W_{\mathrm{pl}}^{\tau}\right)\right|_{\mathrm{R} \approx 250 \mathrm{~nm}}\right.
$$

\section{RESULTS AND DISCUSSION}

This simple model and the use of the LTC allow to capture the size effect on the stability of the Fe-precipitates against SIMT. Fig. 3 shows the experimentally observed number of martensite bands formed in more than 50 Fe-precipitates of diameters from 20 to $110 \mathrm{~nm}$ as reported by Kato et al. [6], together with the predictions from our simulations. The most favorable number of bands $N$ for a given particle size is determined by selecting the microstructure with the smallest stress sufficient to trigger the transformation $\left(\sigma_{\text {eq. }}^{\mathrm{s}}\right)$. In reality, there exists a distribution of lattice orientations of 

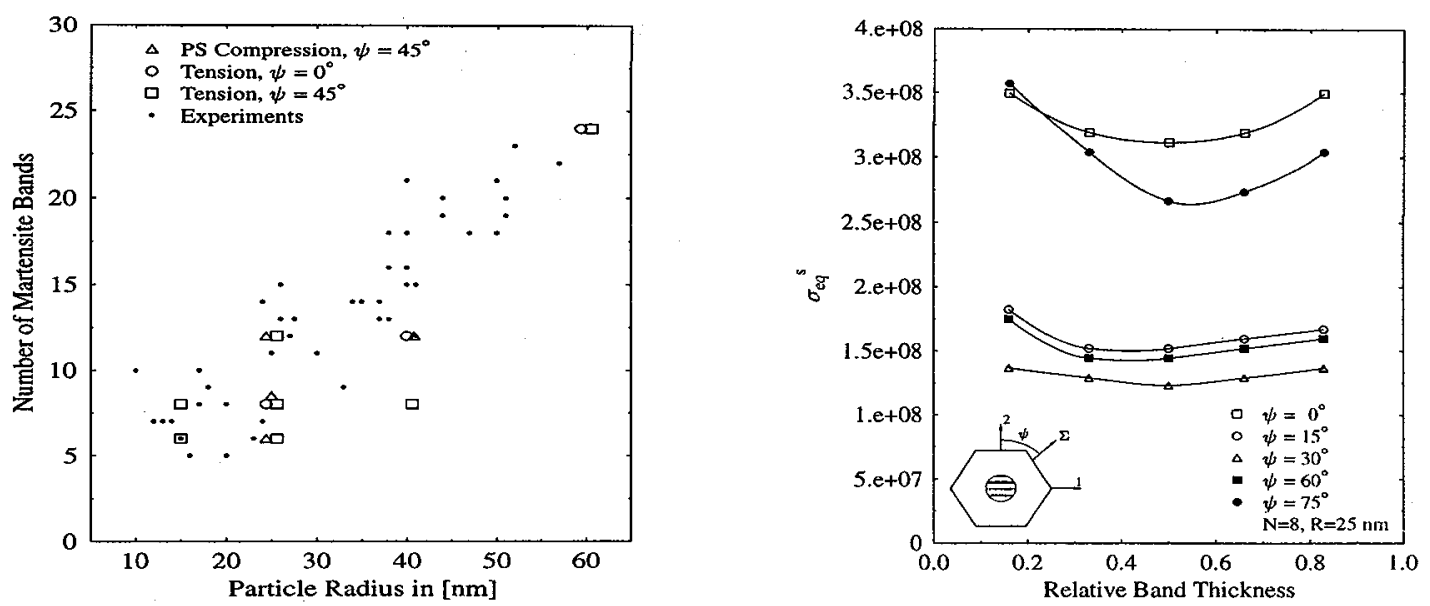

Figure 3: (Left) Size dependence on the number of martensite bands observed in experiments (Kato et al. [6]) and predicted by finite element calculations. (Right) Influence of the relative band thickness $\lambda$ on the onset of transformation in tensile loading for various loading directions.

the Fe-precipitates and thus a distribution of $\psi$. In the simulation we select $\psi=0^{\circ}$ and $\psi=45^{\circ}$ as limiting cases.

One can see from Fig. 3 the influence of the relative band thickness $\lambda$ on $\sigma_{\text {eq. }}^{\mathrm{S}}$ for selected values of $\psi$. For all curves, the particles size is $25 \mathrm{~nm}$, and the number of bands is 8 . If the tensile load is perpendicular to the habit plane $\left(\psi=0^{\circ}\right)$ bands of identical thickness will be formed. However, tilting the direction of the tensile load by an angle of $15^{\circ}$ causes a considerable drop in $\sigma_{\text {eq. }}^{\mathrm{S}}$ and shifts the prediction for $\lambda$ to approximately 0.4 .

Fig. 4 shows the equivalent tensile/compressive stress necessary to trigger the martensitic transformation of the first layer, $\sigma_{\text {eq. }}^{\mathrm{S}}$, as a function of $\psi$, the angle between the tensile load direction and the habit plane normal. Here, the total number of bands is 8 and the particle sizes are 25 and $40 \mathrm{~nm}$. $\psi$ has a marked influence on the stability against SIMT. For $\psi \simeq 45^{\circ}, \sigma_{\text {eq }}^{\mathrm{S}}$ takes on the smallest value, since in this case the shear stresses promoting the SIMT are maximal. Due to the volume dilatation acting in direction $2, \sigma_{\text {eq. }}^{\mathrm{S}}$ is not symmetric about $\psi=45^{\circ}$. For the plane strain compressive loading the same geometry is simulated. In this case the values of $\sigma_{\text {eq. }}^{\mathrm{S}}$ are larger than the ones for tensile loading (Fig. 4). This load type sensitivity stems from the positive volume dilatation associated with the martensitic transformation.

\section{SUMMARY}

The implementation of a thermodynamic transformation criterion into a micromechancial model allows to simulate the strain induced martensitic transformation of small $f c c$-precipitates in a $\mathrm{Cu}-\mathrm{Fe}$ alloy. The influence of the precipitate's size on the resulting martensitic microstructure (number of bands, relative thickness of the bands) is explained by the role of the interface energy and the nonlocal dissipation of the transformation process. The formed martensitic microstructure is very sensitive to the type of load applied. 

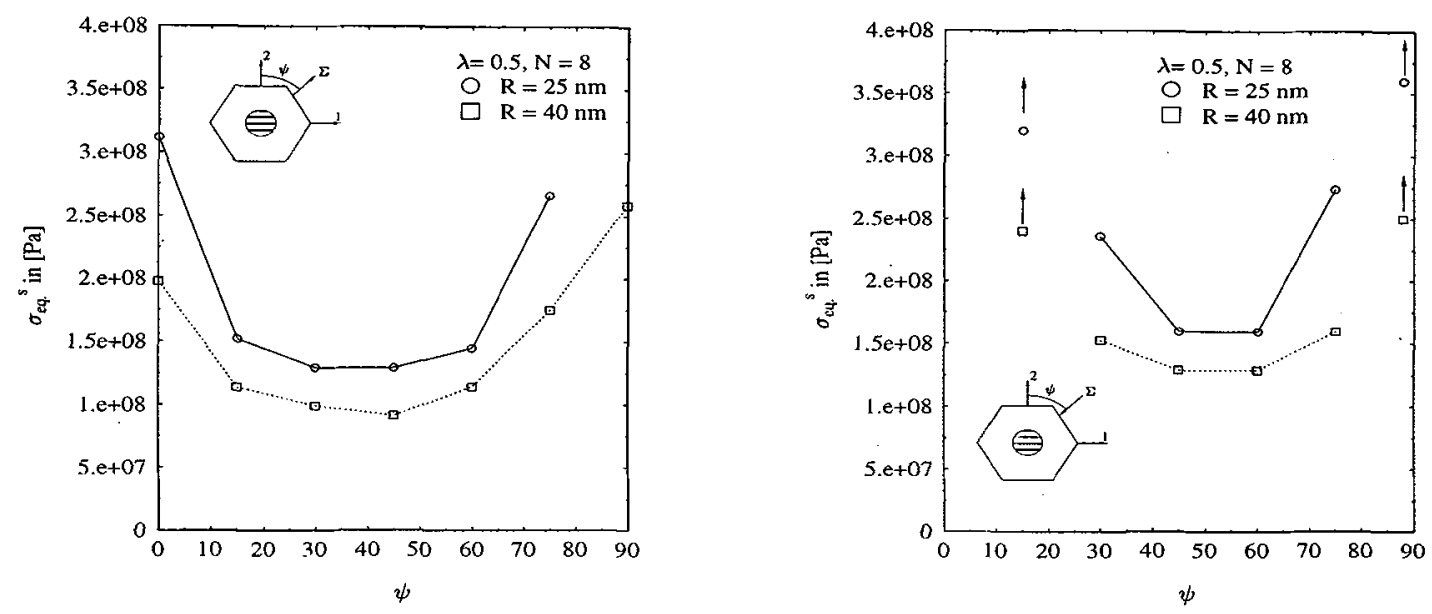

Figure 4: Necessary stress to start the transformation $\left(\sigma_{\text {eq. }}^{\mathrm{S}}\right)$ in the first layer as a function of the angle $\psi$ between the load direction $(\Sigma)$ and the habit plane normal (direction2) predicted for tensile (Left) ant compressive (Right) loading. Results for two particle sizes (25 and $40 \mathrm{~nm}$ ) are shown. The number of martensite bands is 8 .

\section{References}

[1] Easterling, K.E., Miekk-Oja, H. M., Acta metall. 15 (1967) 1133-1141.

[2] Easterling, K. E., Weatherly, G. C., Acta metall. 17 (1969) 845-852.

[3] Matsuura, A., Tsukamoto, M., Watanabe, K., Acta metall. 21 (1973) 1033-1044.

[4] Woolhouse, G. R., Phil. Mag. 28 (1973) 65-83.

[5] Kinsman, K. R., Sprys, J. W., Asaro, R. J., Acta metall. 23 (1975) 1431-1442.

[6] Kato, M., Monzen, R., Mori, T., Acta metall. 26 (1976) 605-613.

[7] Li, C. H., Porter, D. A., Easterling, K. E., Acta metall. 33 (1985) 317-328.

[8] Ishikawa, N., Sato, A., Phil. Mag. A 64 (1991) 387-394.

[9] Hibbit, Karlsson, Sorensen. ABAQUS Version 5.5, Theory Manual, (Hibbit, Karlsson \& Sorensen, Pawtucket, 1995)

[10] Tiefenthaler, B., Reisner, G., Werner, E., Z. Metallkde 86 (1995) 845-851.

[11] Ludwik, P., Elemente der technischen Mechanik, (Julius Springer,

[12] Wechsler, M. S., Lieberman, D. S., Read, T.A., J. Metals 197 (1953) 1503-1515.

[13] Denney, J. M., Acta metall. 4 (1956) 586-592. Berlin, 1907) p. 123.

[14] Fischer, F.D., "Mechanics of Solids with Phase Changes" Berveiller, M., Fischer F. D. Eds., (SpringerVerlag, CISM Courses and Lectures No. 368, chpt.7, to be published in 1997)

[15] Patel, J. R., Cohen, M.,Acta metall. 1 (1953) 531-538.

[16] Nabarro, F.R. N., Theory of crystal dislocations (Oxford University Press, Oxford, 1967) 